\title{
Detection of sibutramine in herbal food supplements by UHPLC/HRMS and UHPLC/MS-MS.
}

\author{
Elina Petkova-Gueorguieva ${ }^{1}$, Kalin Ivanov², Stanislav Gueorguiev ${ }^{1}$, Anna Mihaylova ${ }^{*}$, Vasil \\ Madzharov $^{1}$, Stanislava Ivanova ${ }^{2}$ \\ ${ }^{1}$ Department of Pharmaceutical Sciences, Faculty of Pharmacy, Medical University of Plovdiv, Plovdiv, Bulgaria \\ ${ }^{2}$ Department of Pharmacognosy and Pharmaceutical Chemistry, Faculty of Pharmacy, Medical University of Plovdiv, \\ Plovdiv, Bulgaria \\ ${ }^{3}$ Medical University of Plovdiv, Medical College, Plovdiv, Vasil Aprilov Blvd. 15-A, Bulgaria
}

\begin{abstract}
Recent studies have announced that many food supplements for weight loss contained undeclared sibutramine. We have analysed 10 samples of herbal weight loss food supplements (FS) by HPLC/HRMS and UHPLC/MS-MS. We have established that two of the analysed samples contained sibutramine: respectively $5 \mu \mathrm{g} / \mathrm{per}$ capsule and $20 \mu \mathrm{g} / \mathrm{per}$ capsule. Illegal inclusion of this substance in FS could cause serious side effects and long term health consequences. The regulatory requirements for FS should be enhanced for more comprehensive consumers' protection. The need for mandatory quality control of these products and public awareness is undeniable.
\end{abstract}

Keywords: Sibutramine, HPLC, Food supplements, Analysis, Undeclared substances, WADA. Accepted on July 17, 2018

\section{Introduction}

Sibutramine or 1-(1-(4-chlorophenyl) cyclobutyl)-N, N, 3trimethylbutan-1-amine (Figure 1) is a serotonin-noradrenaline re-uptake inhibitor which was used as a weight loss drug in the recent past [1]. It was approved as an anti-obesity drug in 1997 by FDA and in 1999 by the European Medicines Agency (EMA). It has been established that sibutramine intake increased risk of heart attacks and strokes [2,3]. Since 2010, when EMA concluded that the benefits of sibutramine as weight loss drug do not outweigh its risks, sibutramine drugs have been suspended throughout Europe [1]. Drugs containing sibutramine were withdrawn voluntarily by the manufacturer from the U.S. market in 2010 [2]. Sibutramine has been withdrawn also from the pharmaceutical market of many other countries like: Australia, New Zealand, Canada, China, India, Mexico and others [4-6]. Nowadays there are many cases of undeclared sibutramine in herbal food supplements (FS) for weight loss [7-18]. A variety of techniques could be used successfully for detection of sibutramine: HPLC/MS, HPLCESI-MS, LC-MS/MS and HPTLC. Illegal inclusion of this substance in FS could cause serious health consequences for consumers like non-fatal myocardial infarction and nonfatal stroke [3]. Sibutramine intake is also associated with numerous side effects: agitation; problems with vision, speech, or balance; dry mouth; upset stomach; flu symptoms, insomnia, skin rash and others.

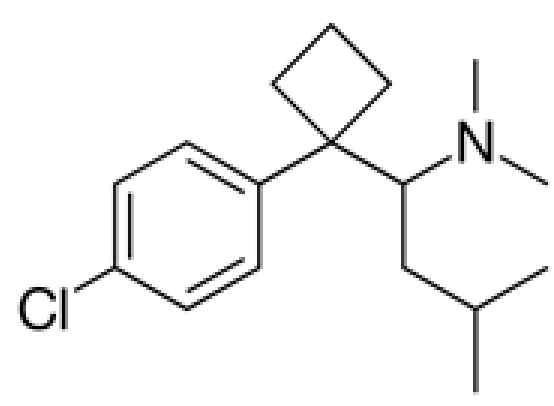

Figure 1. Sibutramine.

\section{Material and Methods}

\section{Materials}

Reference standard: Sibutramine hydrochloride monohydrate with purity $>98 \%$.

Reagents: Acetonitrile for HPLC, (Sigma Aldrich), distilled water.

Samples: 10 herbal food supplements for weight-loss. Samples were purchased from dietary supplement stores, pharmacies and online. 


\section{Methods}

UHPLC/HRMS and UHPLC/MS-MS.

Instrumentation: "Accela UHPLC" with mass spectral detection HRMS "Q-Exative" with H-ESI-interface ("Thermo Fisher Scientific", Waltham, MA, USA) and column EVO C18 $100 \times 3 \mathrm{~mm} 2.6 \mu \mathrm{m}$.; ultrasonic bath (Branson Wilmington, NC, USA); apparatus for ultra-pure water: "MilliQ", "Milipore" (Bedford, MA, USA) and "Elga" (VWR International, Randor, PA, USA).

\section{Sample preparation}

The content of one capsule (or one ground pill) was introduced into a polypropylene $15 \mathrm{ml}$ test-tube and extracted with $10 \mathrm{ml}$, $80 \%$ acetonitrile in water and treated by ultrasound and centrifuging. An aliquot part of the extract was filtered through a $0.45 \mu \mathrm{m}$ membrane filter and the filtrate was diluted 100 -fold with $50 \%$ acetonitrile $/ 50 \%$ water. The obtained extracts of the submitted samples were analyzed up to $3 \mathrm{~h}$ after their preparation.

\section{Mobile phase}

Mobile phase A: $95 \%$ water $/ 5 \%$ acetonitrile $(\mathrm{pH}=4.3 ; 10 \mathrm{~mm}$ ammonium formate).

Mobile phase B: $95 \%$ acetonitrile $/ 5 \%$ water $(\mathrm{pH}=4.3 ; 10 \mathrm{~mm}$ ammonium formate).

Gradient: 50\% mobile phase A/50\% mobile phase B.

Speed: $600 \mu \mathrm{L} / \mathrm{min}$.

\section{Results and Discussion}

According recent studies most customers consider FS as safe products, even safer than conventional drugs. Customers expect no side effects from FS use and many beneficial effects like: slimming or weight-control, increasing immunity and others. Most customers accept FS as pharmaceutical products with high quality. Most customers also trust FS label and the "Health Claims" of these products.

In fact the analytical control for FS is not obligatory. Most researchers and health specialists support that the legislation on FS is liberal and not strict enough. It is undeniable that for the ultimate protection of the consumers, quality control should be applied throughout all processing stages of FS-from the raw material to the finished product [19].

The results from our analyses showed that two of the studied ten FS contained the undeclared ingredient sibutramine-sample No 5 and sample No 8.

Figure 2 presents an extracted chromatogram of $1 \mu 1$ extract of sample No 5 at scanning range of the mass spectrometric detector from 279.68 to $280.68 \mathrm{~m} / \mathrm{z}$ (above) and mass spectrum of the peak with retention time $1.48 \mathrm{~min}$ (below).

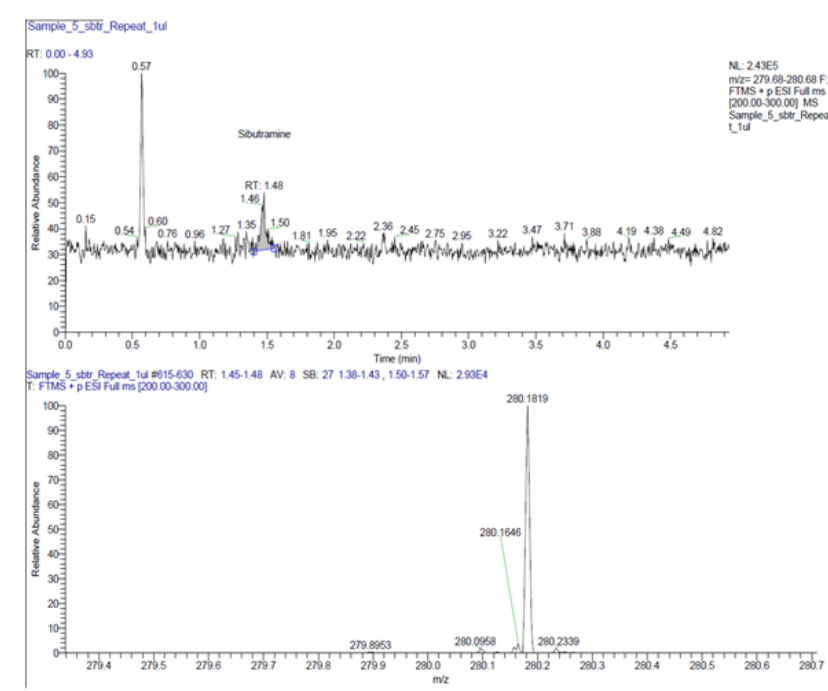

Figure 2. Chromatogram of referent sibutramine and sample No 5 .

Figure 3 presents the data for sample No 5 as a comparison between total ion chromatography in scanning range 200-300 $\mathrm{m} / \mathrm{z}$ (above), extracted chromatogram of the analyte (second line), the electronic signals transmissions $280>125 \mathrm{~m} / \mathrm{z}$ and $280>139 \mathrm{~m} / \mathrm{z}$, characteristic for the analyte (line three and four) and ESI-mass spectrum (below), respectively-on the left-for the referent solution (standard) and on the right-for the analyzed sample (sample No 5).

The retention times and the areas of the respective peaks are marked on the analyte peaks (sibutramine with monoisotopic mass 279.175 Da).

The analytical results enable the conclusion that sample 5 contains $5 \mu \mathrm{g} / \mathrm{per}$ capsule sibutramine.

Figure 4 presents the data for sample No 8 as a comparison between total ion chromatography in scanning range 200-300 $\mathrm{m} / \mathrm{z}$ (above), extracted chromatogram of the analyte (second line), the electronic signals transmissions $280>125 \mathrm{~m} / \mathrm{z}$ and $280>139 \mathrm{~m} / \mathrm{z}$, characteristic for the analyte (line three and four) and ESI-mass spectrum (below), respectively-on the left-for the referent solution (standard) and on the right-for the analyzed sample (sample No 8).

The retention times and the areas of the respective peaks are marked on the analyte peaks (sibutramine with monoisotopic mass 279.175 Da).

The amount of Sibutramine in sample 8 was established to exceed $20 \mathrm{mg}$ per tablet.

Medicinal products containing $10 \mathrm{mg}$ Sibutramine under various trade names have been recommended for once-per-day intake by patients older than $16 \mathrm{y}$. 


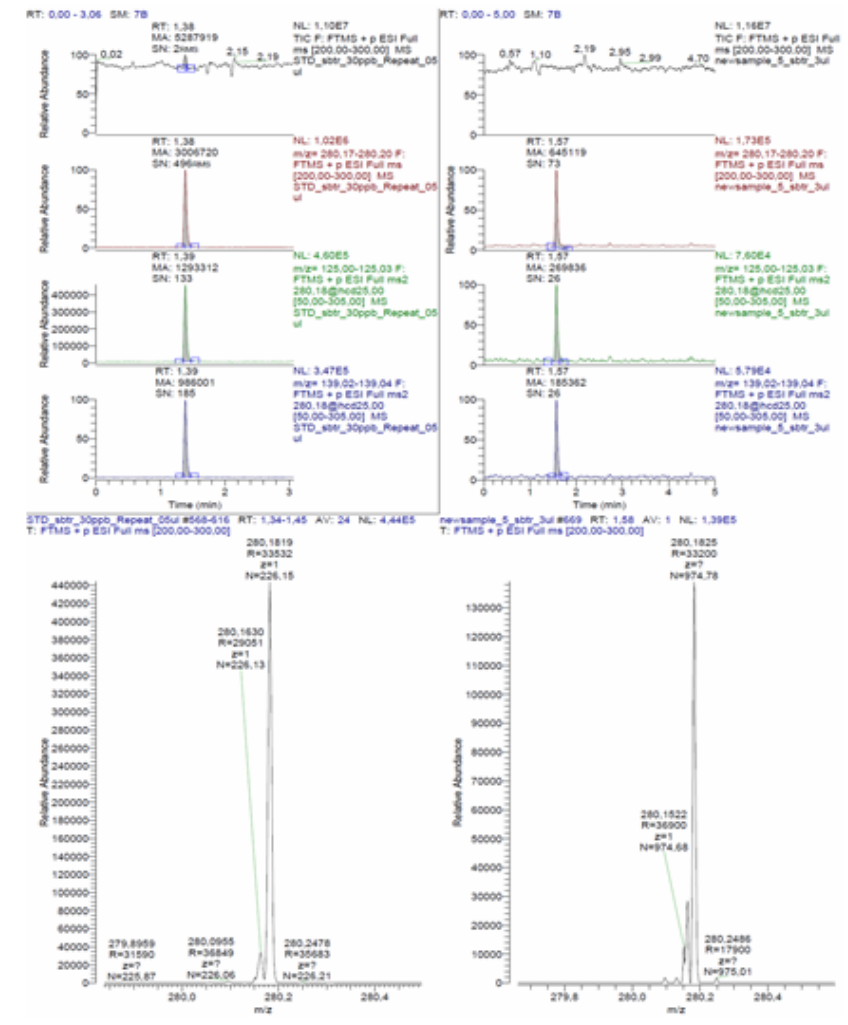

Figure 3. Chromatogram of standard sibutramine (left side) and the analyzed sample No 5 (right side).

The internet commercials for sample 8 state that the product is innovative, specially formulated to help in the weight loss process without forcing the consumers to change their everyday life. Health claims-"the product will reduce the weight with fewer efforts; the effect will be permanent as it acts extremely precisely, in a way that is natural and safe for the organism. Its effect is realized due to a special combination of absolutely neutral synergically bound extracts and herbs.

It suppresses the appetite in a natural way. Recommended intake: in the morning on an empty stomach with a glass of water. Absolutely safe product, without side effects; the food supplement sample No 8 contains an undeclared ingredient in concentration $(>20 \mathrm{mg}$ ), twice greater than the concentration in a drug, containing Sibutramine $(10 \mathrm{mg}$ ) (banned for intake in 2010).

Samples 5 and 8 are extremely hazardous FS and should not be marketed ad FS. The analytical results confirmed the theses of other researchers that numerous food supplements contained undeclared substances.

Globally the studies have revealed that about $20 \%$ of the food supplements contained undeclared substances. It has been established that the declared quantitative and qualitative content frequently did not correspond to the real formulation [20-27].

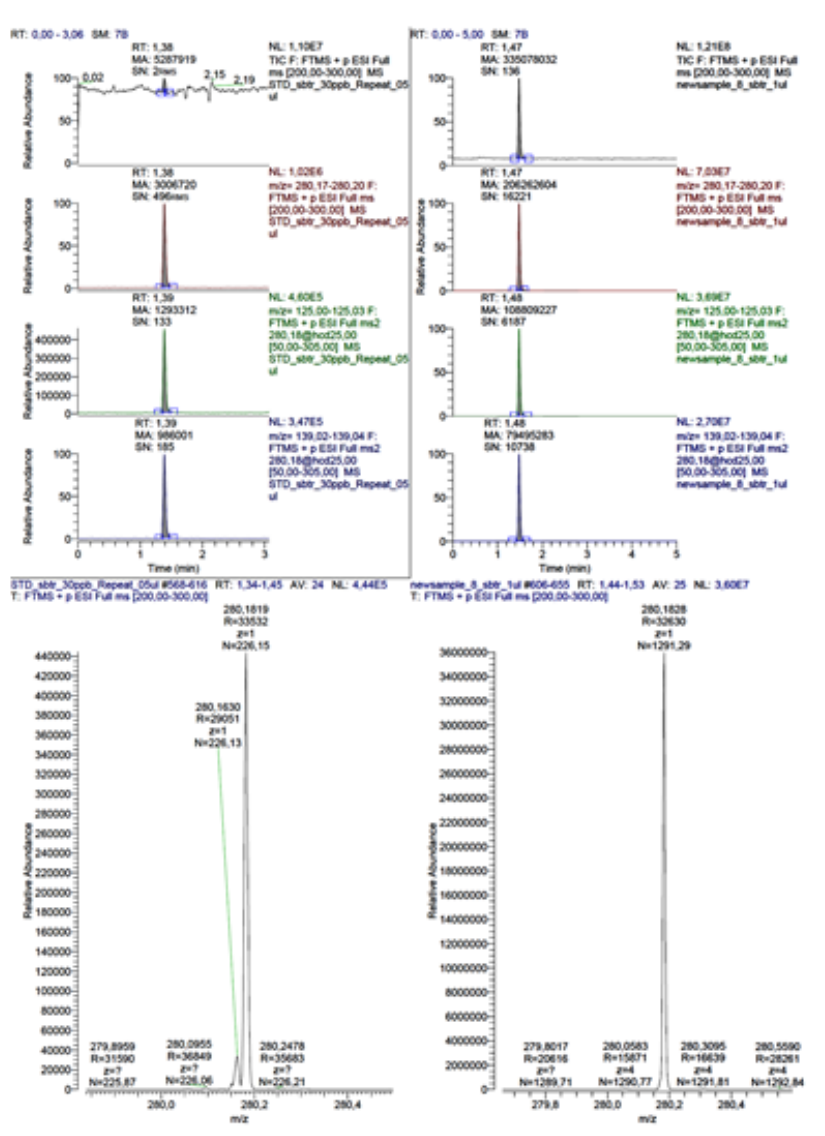

Figure 4. Chromatogram of standard sibutramine and analyzed sample No 8.

\section{Conclusion}

Most customers consider FS as safe products without side effects and contraindications. Customers do not expect a presence of a medicinal product in FS. For a patient with cardiovascular disease, the intake of herbal FS containing undeclared sibutramine could cause serious and even fatal consequences. According to recent studies more than $20 \%$ of herbal FS contain undeclared sibutramine. We have organized a screening of different weight loss FS for sibutramine by UHPLC/HRMS and UHPLC/MS-MS: $20 \%$ of analyzed samples contained undeclared sibutramine. The need for better quality control of FS and public awareness is undeniable.

\section{Acknowledgement}

Medical University Plovdiv, Project Doctorant 2.

\section{References}

1. Science Medicines Health. Sibutramine. Eur Med Agency 2010.

2. Csupor D, Boros K, Dankó B, Veres K, Szendrei K, Hohmann J. Rapid identification of sibutramine in dietary supplements using a stepwise approach. University of Szeged 2013. 
3. James W, Philip T. Effect of sibutramine on cardiovascular outcomes in overweight and obese subjects. New England J Med 2010; 905-917.

4. MEDSAFE. Withdrawal of sibutramine (Reductil) in New Zealand. New Zealand Med Medical Devices Safety Authority 2010.

5. Recalls and Safety Alerts. Update: all marketed sibutramine drugs voluntarily withdrawn in Canada. Government of Canada 2010.

6. Therapeutic Goods Administration. Sibutramine (Reductil)-withdrawal in Australia. Australian Government 2010.

7. Krivohlavek A. Sibutramine in slimming food supplements on the Croatian market determined by validated high-pressure liquid chromatography electrospray tandem mass spectrometry method. J Food Nutr Res 2006; 55: 222.

8. Csupor D. Rapid identification of sibutramine in dietary supplements using a stepwise approach. Pharmazie 2013; 68: $15-18$.

9. Müller D, Wolfgang W, Maren H-C. Chinese slimming capsules containing sibutramine sold over the Internet: a case series. Deutsches Ärzteblatt Int 2009; 106: 218.

10. Zou P. Detection of sibutramine, its two metabolites and one analogue in a herbal product for weight loss by liquid chromatography triple quadrupole mass spectrometry and time-of-flight mass spectrometry. Rapid Communications Mass Spectrometry 2007; 21: 614-618.

11. Kim HJ. Monitoring of 29 weight loss compounds in foods and dietary supplements by LC-MS/MS. Food Additives Contaminants 2014; 31: 777-783.

12. Huang Z. Simultaneous determination of sibutramine and $\mathrm{N}$-di-desmethylsibutramine in dietary supplements for weight control by HPLC-ESI-MS. J Chromatographic Sci 2008; 46: 707-711.

13. Dunn JD. Using a portable ion mobility spectrometer to screen dietary supplements for sibutramine. J Pharm Biomed Analysis 2011; 54: 469-474.

14. Kim JW. Isolation and identification of a sibutramine analogue adulterated in slimming dietary supplements. Food Additives Contaminants 2013; 30: 1221-1229.

15. Petroczi A, Glenn T, Naughton DP. Mission impossible? Regulatory and enforcement issues to ensure safety of dietary supplements. Food Chem Toxicol 2011; 49: 393-402.

16. Reeuwijk NM. Active pharmaceutical ingredients detected in herbal food supplements for weight loss sampled on the Dutch market. Food Additives Contaminants 2014; 31: 1783-1793.

17. Song F. Screening for multiple weight loss and related drugs in dietary supplement materials by flow injection tandem mass spectrometry and their confirmation by liquid chromatography tandem mass spectrometry. J Pharm Biomed Analysis 2014; 88: 136-143.

18. Mathon C. Screening and determination of sibutramine in adulterated herbal slimming supplements by HPTLC-UV densitometry. Food Additives Contaminants 2014; 31: 15-20.

19. Sanzini E. Quality control of plant food supplements. Food Function 2011; 2: 740-746.

20. Ros JJW, Marinus GP, Peter AGMDS. A case of positive doping associated with a botanical food supplement. Pharm World Sci 1999; 21: 44-46.

21. Venhuis BJ, De Kaste D. Towards a decade of detecting new analogues of sildenafil, tadalafil and vardenafil in food supplements: a history, analytical aspects and health risks. J Pharm Biomed Analysis 2012; 69: 196-208.

22. Ernst E. Toxic heavy metals and undeclared drugs in Asian herbal medicines." Trends Pharmacol Sci 2002; 23: 136-139.

23. Martello S, Marialinda F, Marcello C. Survey of nutritional supplements for selected illegal anabolic steroids and ephedrine using LC-MS/MS and GC-MS methods, respectively. Food Additives Contaminants 2007; 24: 258-265.

24. Molinero O, Sara M. Use of nutritional supplements in sports: risks, knowledge, and behavioural-related factors. Nutrición Hospitalaria 2009; 24.

25. Kamber M. Nutrtional supplements as a source for positive doping cases?." Int J Sport Nutrition Exercise Metab 2001; 11: 258-263.

26. Baume N. Research of stimulants and anabolic steroids in dietary supplements. Scandinavian J Med Sci Sports 2006; 16: 41-48.

27. Parr Maria K. Analytical strategies for the detection of non-labeled anabolic androgenic steroids in nutritional supplements. Food Additives Contaminants 2004; 21: 632-640.

\section{*Correspondence to}

Anna Mihaylova

Medical University of Plovdiv

Medical College

Plovdiv

Vasil Aprilov Blvd. 15-A

Bulgaria 